\title{
Congenital Anomaly of Cochlea: A Case Report
}

\author{
Munsif T, Haider S.S, Tewari V, Pasricha N, Siddiqui M S \\ Dept of Anatomy, Era's Lucknow Medical College \& Hospital Lucknow, UP, India \\ Dept of Surgery, Era's Lucknow Medical College \& Hospital Lucknow, UP, India \\ Dept of Anatomy, Era's Lucknow Medical College \& Hospital Lucknow, UP, India \\ Dept of Anatomy, Era's Lucknow Medical College \& Hospital Lucknow, UP, India
}

\begin{abstract}
Congenital malformations of inner ear are rare anomalies. These occur due to arrested development at different stages of embryogenesis.

One rare anomaly of inner ear is Mondini dysplasia. Possibly it occurs due to arrested development of cochlea in its embryonic stage at about sixth week of gestation. In this anomaly only basal turn of cochlea is developed. There is deficient interscalar septum for distal one \& half turn. Thus bony cochlea is restricted to 1.5 turns only. This causes sensorineural hearing loss.

One such anomaly was recognized clinically in a 4yrs old girl who presented with congenital deafness. Thorough systemic \& specific examination was done.Radiological examination (Computed Tomography) revealed, that there was defect in the turn of cochlea i.e. cochlea was only one half turn.
\end{abstract}

Key words: Mondini dysplasia, Cochlea, Sensorineural hearing loss,

\section{Introduction}

Mondini dysplasia [MD] was first described in Latin by Carlo Mondini : Italian anatomist (1729-1803) in 1791[1]. Mondini dysplasia is a type of inner ear malformation that is present at birth (congenital). Individuals with Mondini dysplasia have one and a half coils of the cochlea instead of the normal two coils[2]. It may occur in one ear (unilateral) or both ears (bilateral) and can cause varying degrees of sensorineural hearing loss, although most individuals have profound hearing loss[2].The condition can also predispose to recurrent meningitis[3]. It is caused by disruption in the embryonic development of the inner ear during the seventh week of gestation[3]. The condition may be isolated (occur with no other conditions or malformations) or may occur with other ear malformations or a number of syndromes[4]. Treatment options may include surgical repair of the defect to prevent recurrent meningitis; amplification aids for those with residual hearing; and cochlear implantation [5]. Development of inner ear begins early during embryogenesis. By the end of eighth week, the membranous labyrinth assumes its characteristic convoluted shape. Gradual ossification of the otic capsule develops around the membranous labyrinth and is essentially complete by birth.

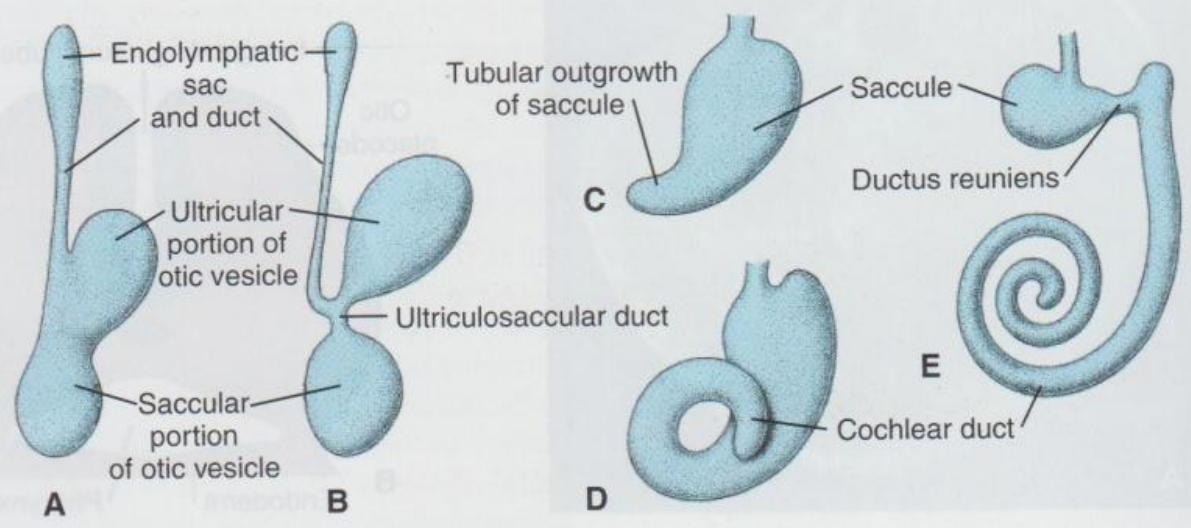

Fig- 1 A,B shows- Development of otocyst showing dorsal utricular portion with endolymphaticduct and ventral sacculer portion. $\mathrm{C}, \mathrm{E}$ - Cochlear duct at 6,7,8 week of development

Most inner ear malformations arise when formation of the membranous labyrinth is interrupted during the first trimester of pregnancy. This interruption may be either a result of inborn genetic error or a consequence of teratogenic exposure during period of inner ear organogenesis between $4^{\text {th }}-8^{\text {th }}$ week of gestation One rare anomaly of inner ear is Mondini dysplasia. It occurs due to arrested development of cochlea in its embryonic 
stage at about sixth week of gestation.In this anomaly only basal turn of cochlea is developed. There is deficient interscalar septum for the distal one \& half turn. Thus bony cochlea is restricted to 1.5 turns only. This causes sensorineural hearing loss. The normal cochlea has two and one-half turns. A cochlear malformation consists of a membranous abnormality, a bony abnormality, or a combination of these two. If cochlear development is arrested in the embryo, a common cavity may occur instead of the snail like cochlea [7]. It is clear from Hartley's translation that the inner ear anomaly described by Mondini consists of (1) a cochlea of one-and-onehalf turns instead of the normal two-and-one-half turns, comprising a normal basal turn and a cystic apex in place of the distal one-and-one-half turns; (2) an enlarged vestibule with normal semicircular canals; and (3) an enlarged vestibular aqueduct containing a dilated endolymphatic sac. Thus, the cochlear anomaly was relatively mild, and would correspond to incomplete partition in the spectrum of congenital cochlear malformation because of an arrest of embryogenesis, as proposed by Jackler et al [6]. Arrest at earlier stages in the spectrum include, cochlear hypoplasia, common cavity and cochlear aplasia. Even earlier arrests result in otocyst and complete labyrinthine aplasia (Michel's anomaly) [6].

\section{Case Report}

A 4 year old girl was referred for evaluation for a possible cochlear implant by her primary care physician. She was initially evaluated for congenital hearing loss at the age of 10 months due to delay in speech development. Brain-stem evoked-response audiometry at the age of 10 months showed profound hearing loss in both ears with no response to click audiometry at maximal stimulation (100dB). There was no history of maternal infections or drug use during pregnancy. Patient's mother was under regular prenatal care by a qualified gynaecologist and had received adequate vitamin A supplements. The patient was born during an uneventful spontaneous vaginal delivery and had remained stable after birth. She received immunisations according to local recommendations. All her developmental milestones were up to the mark except hearing/speech. On examination, she appeared in good overall health. She was responsive to visual and tactile stimuli; however she did not respond to auditory stimuli. She had appropriate developmental milestones other than speech. Otological examination revealed no apparent deformities of the external or middle ear structures. She was planned for a cochlear implant.Computed tomography (CT) scan revealed non-visualization of apical and basal turns of cochlea on left side.

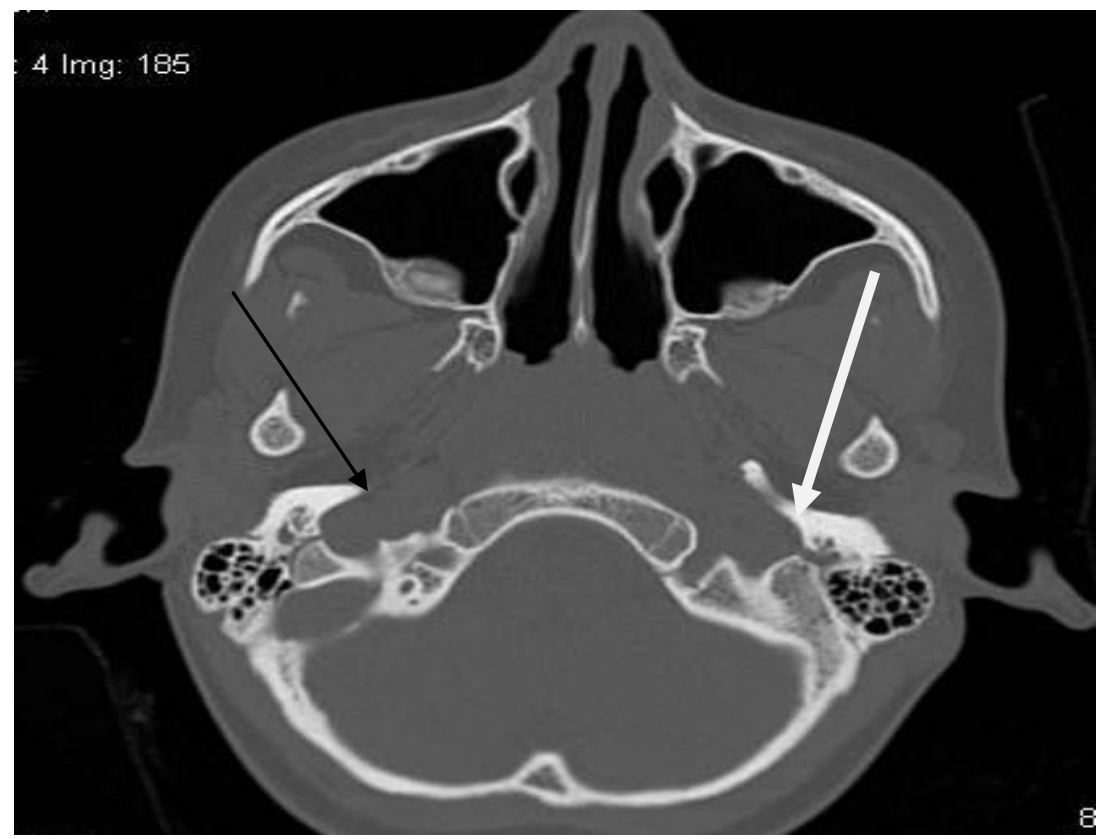

Fig-1 CT Temporal bone ( For cochlea ) shows normal cochlea on rt side black arrow non visualization of apical \& basal turns left white arrow 


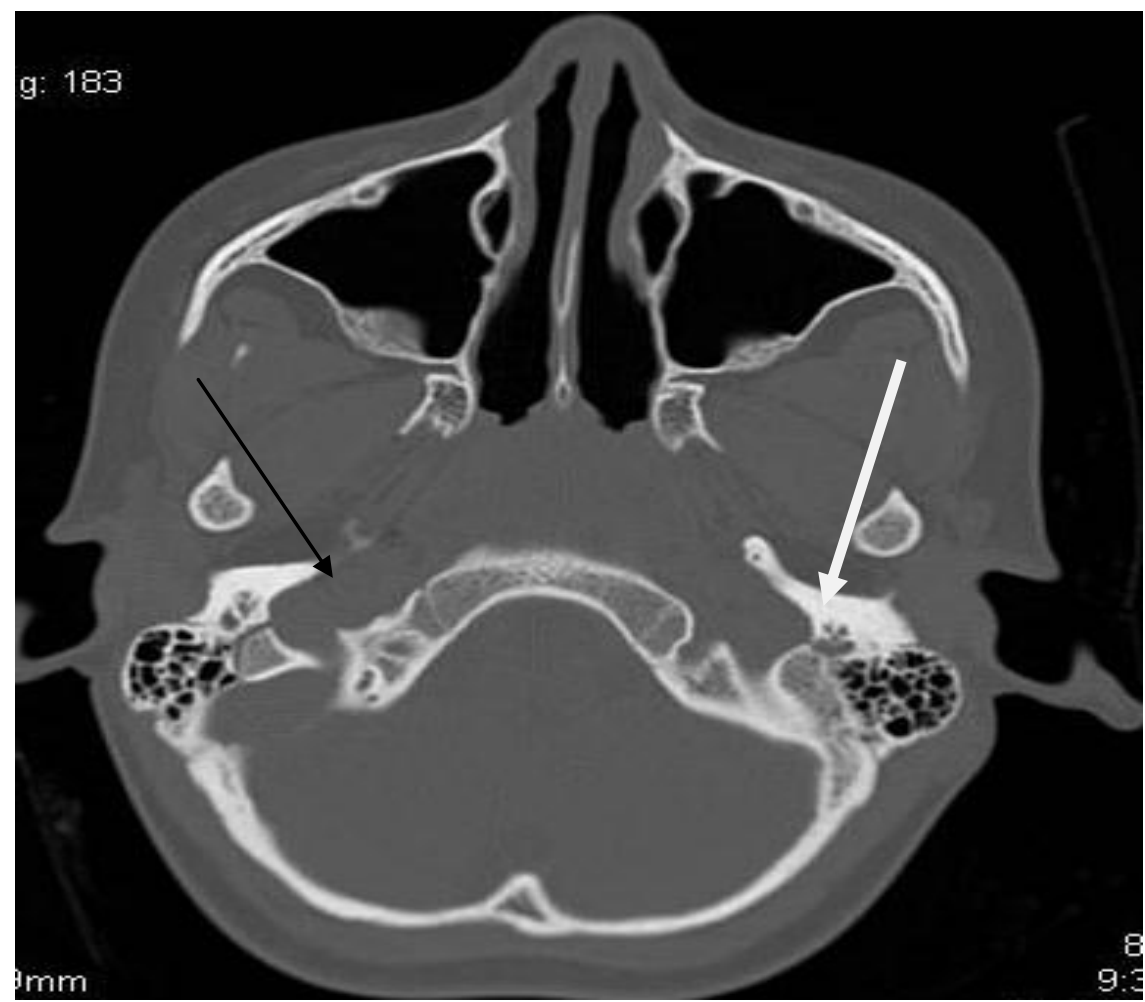

Fig-2 CT Temporal bone normal cochlea rt black arrow non visualization of cochlea left white arrow

\section{Discussion}

Cochlea is prone to variety of congenital anomalies depending on exact time at which an insult occurs during embryogenesis. A classification first proposed in 1987 by Jacker et al [6], has become widely accepted which divides congenital cochlear anomalies according to timing of developmental arrest. Starting from the $3^{\text {rd }}$ week of gestation, an insult during each subsequent week results in a distinct inner ear abnormality.

$3^{\text {rd }} \mathrm{wk}$ - complete labyrinthine aplasia

$4^{\text {th }} \mathrm{wk}-$ common cavity of cochlea \& vestibule

$5^{\text {th }}$ wk - cochlear aplasia

$6^{\text {th }}$ wk - cochlear hypoplasia

$7^{\text {th }}$ wk - incomplete partition \& dilatation defects (Mondini dysplasia)

Underlying cause of Mondini Dysplasia in most individuals appears to remain unclear. It has also been suggested that Mondini dysplasia may be associated with substance that may harm a developing fetus when a pregnant women is exposed to teratogens such as Thalidomide, Rubella, cytomegalo virus etc [7]. Some suggested Retinoids (Vit A) or other factors to which fetus may be exposed to early in pregnancy may have contributed to some cases of isolated Mondini dysplasia [4]. Developmental damage to cochlear structure and function is not restricted to agents that cause gross structural malformations. Certain ototoxic drugs like Aminoglycosides antibiotics if administered in $1^{\text {st }}$ trimester of pregnancy may cause hearing loss. The risk is not restricted to the first trimester. Even at low doses, hearing losses were seen when aminoglycoside antibiotics were administered during the last two trimesters of pregnancy, which has obvious implications for use of these drugs in pregnant women and in premature infants who have not yet reached functional cochlear maturity. [6] Recently a type of mutation called a microdeletion (a tiny loss of genetic material on a chromosome that may span several genes) involving the POU3F4 gene on the $\mathrm{X}$ chromosome was detected in some individuals with familial MD[5].

Relative incidence of cochlear malformation reported by Jacker et al 1987-

Incomplete partition Mondini 55\%, Common cavity 26\%, Chochlear hypoplasia 15\%, Cochlear aplasia 3\%, Complete labyrinthine aplasia (Michels) $1 \%$

Associations - Mondini dysplasia has been associated with thalidomide and rubella embryopathies as well as a number of syndromes: Syndromes that have been associated with MD include Klippel Feil syndrome, Pendred syndrome, DiGeorge syndrome \& some chromosomal trisomies[2]. 
Use of cochlear implants to treat patients with inner ear malformations such as Mondini dysplasia has been increasingly successful. Various results of cochlear implantation in individuals with Mondini dysplasia have been reported in the literature.[8]

\section{Conclusion}

In present case report, we report a girl child with the history of impaired hearing. CT-scan revealed Mondini malformation. Mondini dysplasia is a kind of congenital malformation of the osseous labyrinth which can be diagnosed by radiographic examination. The ability of CT-scan in evaluation of temporal bone, makes it the best choice for examination as it needs less than one minute immobilization. Mondini dysplasia is listed as a rare disease by the office of rare diseases (ORD) of National Institute of Health(NIH). It effects less than 200,000 people in US population. This type of malformation has been described as flattened cochlea with development of only basal coil. The prognosis of Mondini deformity varies. Case presented is related to anatomical deformities of cochlea.

\section{References}

[1]. Mondini C. Anatomia surdi nati sectio: De Bononiensi Scientiarum et Artium Institute atque Academia commentarii. . Bononiae. $1791 ; 7: 419-428$.

[2]. Masri A. et al. Mondini malformation associated with diastematomyelia and presenting with recurrent meningitis. J Child Neurol. May 2011; 26(5):622-624.

[3]. Kamata A, Obinata K, Kon N, Suganuma H, Niizuma T, Kinoshita K. Recurrent meningitis with Mondini dysplasia after the operation and vaccination. Pediatr Int. April 2010.

[4]. Huang S. et al. Extremely discrepant mutation spectrum of SLC26A4 between Chinese patients with isolated Mondini deformity and enlarged vestibular aqueduct. J Transl Med. September 2011; 9:167.

[5]. Tullu MS, Khanna SS, Kamat JR, Kirtane MV. Mondini dysplasia and pyogenic meningitis. Indian J Pediatr. July 2004; 71(7):655657.

[6]. Jackler RK, Luxford WM, House WF. Congenital malformations of the inner ear: a classification based on embryogenesis. Laryngoscope. 1987;97 (3 Pt 2 Suppl 40): 2-14.

[7]. Timothi C HAIN,MD Chicago Dizziness \& Hearing,Mondini \& Michel malformation of inner ear.

[8]. Daneshi A, Hassanzadeh S, Abasalipour P, Emamdjomeh H, Farhadi M. Cochlear implantation in Mondini dysplasia. ORL J Otorhinolaryngol Relat Spec. January-February 2003. 